\title{
Phasing Strategies II - Molecular Replacement
}

Paul D. Adams ${ }^{1}$, Pavel V. Afonine ${ }^{1}$, Thomas C. Terwilliger ${ }^{2}$, Rob D. Oeffner ${ }^{3}$, Airlie J. $\mathrm{McCoy}^{3}$, Randy J. Read ${ }^{3}$

${ }^{1}$ Molecular Biophysics \& Integrated Bioimaging Division, Lawrence Berkeley National Laboratory, Berkeley, CA 94720-8235, USA

${ }^{2}$ Los Alamos National Laboratory, Los Alamos, NM 87545-0001, USA

${ }^{3}$ Department of Haematology, University of Cambridge, Cambridge Institute for Medical Research, Wellcome Trust/MRC Building, Cambridge CB2 OXY, England

E-mail: $\underline{\text { PDAdams@,lbl.gov }}$

The phase problem still remains a challenge in X-ray crystallography. The growth of the database of known structures often now makes it possible to solve the phase problem without resorting to experimental phasing methods. In this molecular replacement method the problem is solved by calculating phases from a similar structure placed in the position of the unknown molecule in the crystallographic unit cell. The algorithms underlying the method will be discussed along with recent developments designed to address challenging molecular replacement cases. 